PROCEEDINGS OF THE AMERICAN MATHEMATICAL SOCIETY

Volume 124, Number 10, October 1996

\title{
FIXED POINTS OF APPROXIMABLE MAPS
}

\author{
SEHIE PARK
}

(Communicated by Palle E. T. Jorgensen)

\begin{abstract}
We present a simple proof of the Leray-Schauder type theorem for approximable multimaps given recently by Ben-El-Mechaiekh and Idzik. We apply this theorem to obtain a Schaefer type theorem, the Birkhoff-Kellogg type theorems, a Penot type theorem for non-self-maps, and quasi-variational inequalities, all related to compact closed approximable maps.
\end{abstract}

\section{INTRODUCTION}

Recently, Ben-El-Mechaiekh and Idzik [BI] derived a Leray-Schauder type theorem for approximable multimaps from a matching theorem of Ky Fan. We present, in this paper, a simple proof of their theorem using an earlier fixed point theorem due to Ben-El-Mechaiekh et al. Moreover, we apply their theorem to obtain a Schaefer type theorem, the Birkhoff-Kellogg type theorems, a Penot type theorem for non-self-multimaps, and quasi-variational inequalities with respect to compact closed approximable maps. Finally, we indicate that our method works also for other classes of maps including composites of acyclic maps.

A t.v.s. means a Hausdorff topological vector space. Int, Bd, - , and co denote the interior, boundary, closure, and convex hull, respectively.

For subsets $X$ and $Y$ of t.v.s. $E$ and $F$, respectively, a multimap or map $\Phi$ : $X \multimap Y$ is a function from $X$ into the power set of $Y$ with nonempty values. $\Phi$ is said to be closed if it has a closed graph $\operatorname{Gr}(\Phi) \subset X \times Y$, and compact if its range $\Phi(X)$ is contained in a compact subset of $Y$.

Given two open neighborhoods $U$ and $V$ of the origin 0 of $E$ and $F$, respectively, a $(U, V)$-approximative continuous selection of $\Phi$ is a continuous function $s: X \rightarrow Y$ satisfying

$$
s(x) \in(\Phi[(x+U) \cap X]+V) \cap Y \text { for } \quad x \in X .
$$

A map $\Phi: X \multimap Y$ is said to be approximable if its restriction $\left.\Phi\right|_{K}$ to any compact subset $K$ of $X$ admits a $(U, V)$-approximative continuous selection for every $U$ and $V$ as above.

Received by the editors March 30, 1995.

1991 Mathematics Subject Classification. Primary 47H10, 54C60; Secondary 54H25, 49J35, 49K35, 52A07, 55M20.

Key words and phrases. Hausdorff topological vector space (t.v.s.), multimap (map), $(U, V)$ approximative continuous selection, approximable, closed map, compact map, theorems of LeraySchauder type, Schaefer type, Birkhoff-Kellogg type, quasi-variational or variational inequalities.

Supported in part by KOSEF-951-0102-006-2

(C)1996 American Mathematical Society 
For properties and examples of approximable maps, we refer to [BI] and references therein.

\section{A Leray-Schauder TyPe THEOREM}

We begin with the following particular form of [P2, Theorem 3]:

Theorem 1. Let $X$ be a convex subset of a locally convex t.v.s. E and $\Phi: X \multimap X$ a compact closed approximable map. Then $\Phi$ has a fixed point $\hat{x} \in X$; that is, $\hat{x} \in \Phi(\hat{x})$.

A slightly particular version of Theorem 1 appeared in [Be2, Theorem 2.4], [BD2, Corollary 3.4], [BD3, Corollary 7.3]. In case $X$ itself is compact, Theorem 1 is given in [BD2, Corollary 3.6], [BD3, Corollary 7.6].

From Theorem 1, we give a simple proof of the following theorem due to BenEl-Mechaiekh and Idzik [BI]:

Theorem 2. Let $X$ be a closed subset of a locally convex t.v.s. E such that $0 \in$ Int $X$ and $\Phi: X \multimap E$ a compact closed approximable map. Then either

(1) $\Phi$ has a fixed point; or

(2) $\lambda x \in \Phi(x)$ for some $\lambda>1$ and $x \in \operatorname{Bd} X$.

Proof. Let $R \subset X$ be defined by

$$
R=\{x \in X: x \in t \Phi(x) \text { for some } t \in[0,1]\},
$$

which is nonempty since $0 \in R$. Moreover, it is closed since $\Phi$ is closed. Therefore, $R$ is compact since $\Phi$ is compact.

Suppose that

(LS) $\Phi(y) \cap\{\lambda y: \lambda>1\}=\emptyset$ for all $y \in \operatorname{Bd} X$.

Then $R \cap \operatorname{Bd} X=\emptyset$. Since $X$ is completely regular, there exists a continuous function $r: X \rightarrow[0,1]$ such that $r(x)=1$ for $x \in R$ and $r(x)=0$ for $x \in \operatorname{Bd} X$.

Let $\Psi: E \multimap E$ be defined by

$$
\Psi(x)=\left\{\begin{array}{lll}
r(x) \Phi(x) & \text { if } & x \in X \\
\{0\} & \text { if } & x \notin X .
\end{array}\right.
$$

Since $\Phi$ is compact and closed, so is $\Psi$. Moreover, $\Psi$ is approximable. In fact, for any compact subset $K$ of $X$, if $s: K \rightarrow E$ is a $(U, V)$-approximative continuous selection of $\left.\Phi\right|_{K}$, then $r s: K \rightarrow E$ is a $(U, V)$-approximative continuous selection of $\left.\Psi\right|_{K}$. Therefore, $\Psi$ has a fixed point $x \in E$ by Theorem 1 . Since $0 \in \operatorname{Int} X \subset X$, if $x \notin X$ and $x \in \Psi(x)=\{0\}$, we have a contradiction. Hence, $x \in X$. Now $x \in \Psi(x)$ implies $x \in R$ and $r(x)=1$. Therefore, $x \in \Phi(x)$. This completes our proof.

Remarks. 1. We followed the method of Schöneberg [Sö]. Theorem 2 includes [Be1, Theorem 5] and many others. See [BI], [P4].

2. In a sense, Theorem 2 is more general than Theorem 1. As Ben-El-Mechaiekh [Be1, p. 314] noted, Theorem 2 works for a self-map $\Phi: X \multimap X$ where $X$ is starshaped with the star-center $0 \in \operatorname{Int} X$. Moreover, Theorem 2 also works for a selfmap $\Phi: \bar{X} \rightarrow \bar{X}$ where $X$ is a shrinkable subset; that is, $[0,1)(\overline{X-p}) \subset \operatorname{Int}(X-p)$ for some $p \in X$. See Klee $[\mathrm{K}]$. 


\section{A SCHAEFER TYPE THEOREM}

From Theorem 2, we have the following :

Theorem 3. Let $E$ be a locally convex t.v.s. and $\Phi: E \multimap E$ a compact closed approximable map. Then either

(1) $\Phi$ has a fixed point; or

(2) the set $A=\{x \in E: x \in t \Phi(x)$ for some $t \in(0,1)\}$ is not bounded.

Proof. Suppose that $A$ is bounded. Let $X$ be a bounded neighborhood of 0 such that $A \subset \operatorname{Int} X$. Then no $y \in \operatorname{Bd} X$ satisfies $\lambda y \in \Phi(y)$ for any $\lambda>1$. Therefore, by Theorem $2, \Phi$ has a fixed point in $\bar{X}$.

Remark. Theorem 3 was first obtained by Schaefer [Sc1, Sc2] for a completely continuous map $f: E \rightarrow E$ on a complete locally convex t.v.s. $E$.

\section{The Birkhoff-Kellogg type theorems}

As an application of Theorem 2, we have the following generalization of the Birkhoff-Kellogg theorem [BK].

Theorem 4. Let $X$ be a closed subset of a locally convex t.v.s. E such that $0 \in$ Int $X$, and $\Phi: X \multimap E$ a compact closed approximable map such that $\lambda \Phi(X) \cap X=\emptyset$ for some $\lambda$. Then $\left.\Phi\right|_{\operatorname{Bd} X}$ has an eigenvalue; that is, $\mu x \in \Phi(x)$ for some $\mu \neq 0$ and $x \in \operatorname{Bd} X$.

Proof. Note that $\lambda \neq 0$ and $\lambda \Phi: X \multimap E$ is a compact closed approximable map. Moreover, $\lambda \Phi$ has no fixed point. Therefore, by Theorem 2, there exist $x \in \operatorname{Bd} X$ and $\mu>1$ such that $\mu x \in \lambda \Phi(x)$, whence we have $\left(\lambda^{-1} \mu\right) x \in \Phi(x)$, where $\lambda^{-1} \mu \neq 0$. This completes our proof.

Remark. If $\lambda>0$ in Theorem 4, then $\left.\Phi\right|_{\operatorname{Bd} X}$ has an invariant direction (a positive eigenvalue); that is, $\mu x \in \Phi(x)$ for some $\mu>0$ and $x \in \operatorname{Bd} X$.

From Theorem 4, we obtain

Theorem 5. Let $S$ be the unit sphere of a normed vector space $E$ of infinite dimension, and $\Phi: S \multimap E$ a compact closed approximable map such that $0 \notin \overline{\Phi(S)}$. Then $\Phi$ has an invariant direction.

Proof. Since $E$ is infinite dimensional, by the Dugundji extension theorem, there exists a retraction $r: E \rightarrow S$ such that $r(x)=x /\|x\|$ if $\|x\| \geq 1$ and $\|r(x)\|=1$ if $\|x\| \leq 1$. Let $\Psi=\Phi r: E \multimap E$. Then $\Psi$ is a compact closed approximable map. Let $B$ be the closed unit ball. Then $\lambda \Psi(B) \cap B=\emptyset$ for some $\lambda>0$ since $\Psi(B) \subset \overline{\Phi(S)}$ and $0 \notin \overline{\Phi(S)}$. Therefore, by Theorem 4 with $X=B,\left.\Psi\right|_{S}$ has an eigenvalue. Since $\lambda>0$, this eigenvalue is positive. This completes our proof.

Theorem 5 reduces immediately to the following fixed point theorem:

Theorem 6. Let $S$ be the unit sphere of a normed vector space $E$. Then $E$ is of infinite dimension if and only if any compact closed approximable map $\Phi: S \multimap S$ has a fixed point. 


\section{FiXed POINTS OF NON-SELF-MAPS}

Combining Theorems 1 and 2, we obtain the following fixed point theorem for approximable maps:

Theorem 7. Let $X$ be a closed convex subset of a locally convex t.v.s. E, and $\Phi: X \multimap E$ a compact closed approximable map. If $\Phi(\operatorname{Bd} X) \subset X$, then $\Phi$ has a fixed point.

Proof. If Int $X=\emptyset$, then $X=\operatorname{Bd} X$ and $\Phi: X \multimap X$ has a fixed point by Theorem 1. If Int $X \neq \emptyset$, then we may assume $0 \in \operatorname{Int} X$. Now for each $x \in \operatorname{Bd} X, \Phi(x) \subset X$ implies $\Phi(x) \cap\{\lambda x: \lambda>1\}=\emptyset$ since $X$ is shrinkable; that is, (LS) holds. Therefore, by Theorem 2, $\Phi$ has a fixed point.

Remark. For a compact closed map $\Phi: X \multimap E$ with convex values, Theorem 7 reduces to Penot [Pe, Proposition 1.4], which contains the particular case for a single-valued continuous map due to Brezis. See [Pe].

\section{Quasi-VARIATIONAL OR VARIATIONAL INEQUalities}

From Theorem 7, we have the following quasi-variational inequality :

Theorem 8. Let $X$ be a closed convex subset of a locally convex t.v.s. E, Y a compact subset of $E$, and $f: X \times Y \rightarrow \mathbf{R}$ an u.s.c. function. Let $T: X \multimap Y$ be a closed map such that $T(\operatorname{Bd} X) \subset X \cap Y$. Suppose that

(i) the function $M$ defined on $X$ by

$$
M(x)=\sup _{y \in T(x)} f(x, y) \quad \text { for } \quad x \in X
$$

is l.s.c.; and

(ii) the map $\Phi: X \multimap Y$ defined on $X$ by

$$
\Phi(x)=\{y \in T(x): f(x, y)=M(x)\} \quad \text { for } \quad x \in X
$$

is approximable.

Then there exists an $\hat{x} \in X$ such that

$$
\hat{x} \in T \hat{x} \quad \text { and } \quad f(\hat{x}, \hat{x})=M(\hat{x}) .
$$

Proof. Note that the marginal function $M$ in (i) is actually continuous since $f$ is u.s.c. and $T$ is a compact-valued u.s.c. map, by the well-known result of Berge [Br]. Now, each $\Phi(x)$ is nonempty. Moreover, $\Phi$ is a closed map. In fact, let $\left(x_{\alpha}, y_{\alpha}\right) \in \operatorname{Gr}(\Phi)$, the graph of $\Phi$, and $\left(x_{\alpha}, y_{\alpha}\right) \rightarrow(x, y)$ in $X \times Y$. Then

$$
\begin{aligned}
f(x, y) & \geq \varlimsup_{\alpha} f\left(x_{\alpha}, y_{\alpha}\right)=\varlimsup_{\alpha} M\left(x_{\alpha}\right) \\
& \geq \varlimsup_{\alpha} M\left(x_{\alpha}\right) \geq M(x)
\end{aligned}
$$

and, since $\operatorname{Gr}(T)$ is closed in $X \times Y, y_{\alpha} \in T\left(x_{\alpha}\right)$ implies $y \in T(x)$. Hence $(x, y) \in$ $\operatorname{Gr}(\Phi)$. Therefore, $\Phi: X \multimap E$ is a compact closed approximable map satisfying $\Phi(\operatorname{Bd} X) \subset T(\operatorname{Bd} X) \subset X$. Hence, by Theorem $7, \Phi$ has a fixed point $\hat{x} \in X$; that is, $\hat{x} \in T \hat{x}$ and $f(\hat{x}, \hat{x})=M(\hat{x})$. This completes our proof.

Remark. If $T: X \multimap Y \subset X$, then we can obtain Theorem 8 from Theorem 1 without assuming the closedness of $X$. In this case, Theorem 8 is actually equivalent to Theorem 1. Moreover, in this case, Theorem 8 extends Takahashi [T, Theorem 4], which was applied to prove Fan's generalizations of fixed point theorems of Schauder and Tychonoff. 
The following is an immediate consequence of Theorem 8.

Theorem 9. Let $X$ be a compact convex subset of a locally convex t.v.s., $f: X \times$ $X \rightarrow \mathbf{R}$ a continuous function such that all of the sets

$$
\left\{y \in X: f(x, y)=\inf _{y \in X} f(x, y)\right\}
$$

for $x \in X$ are (1) convex, (2) contractible, (3) decomposable, or (4) $\infty$-proximally connected. Then there exists an $\hat{x} \in X$ such that

$$
f(\hat{x}, \hat{x}) \leq f(\hat{x}, y) \quad \text { for all } \quad y \in X .
$$

Proof. In any case (1)-(4), the map $\Phi: X \multimap X$ defined by

$$
\Phi(x)=\left\{y \in X: f(x, y)=\inf _{y \in X} f(x, y)\right\} \quad \text { for } \quad x \in X
$$

is a compact closed approximable map. See [BI]. Now, the conclusion follows from Theorem 8 by putting $X=Y$ and $T(x)=X$ for all $x \in X$.

Remark. As in [PC], Theorem 9 can be used to obtain variational or variational-like inequalities due to Hartman-Stampacchia, Browder, Lions-Stampacchia, Mosco, Juberg-Karamardian, Park, Karamardian, Parida-Sahoo-Kumar, Behera-Panda, and Siddiqi-Khaliq-Ansari. For the literature, see [PC].

Finally the approximable map in Theorem 1 can be replaced by an acyclic map [P1, Theorem 7(iii)], a composite of acyclic maps [PSW, Theorem 2(iii)], or a composite of admissible maps [P2, Theorem 3(iii)]. Therefore, following our method, Theorems 2-9 also hold for these classes of maps. In fact, we already have Theorem 8 for acyclic maps [P3, Theorem 2] and for admissible maps [PC, Theorem 2]. Hence, as was noted in $[\mathrm{BI}]$, the set in Theorem 9 can be acyclic.

\section{REFERENCES}

[Be1] H. Ben-El-Mechaiekh, A remark concerning a matching theorem of Ky Fan, Chinese J. Math. 17 (1989), 309-314. MR 90m:47071

[Be2] - General fixed point and coincidence theorems for set-valued maps, C. R. Math. Rep. Acad. Sci. Canada 13 (1991), 237-242. MR 92m:47110

[BD1] H. Ben-El-Mechaiekh and P. Deguire, Approximation of non-convex set-valued maps, C. R. Acad. Sci. Paris 312 (1991), 379-384. MR 92d:54058

[BD2] General fixed point theorems for non-convex set-valued maps, C. R. Acad. Sci. Paris 312 (1991), 433-438. MR 92d:54059

[BD3] Approachability and fixed points for non-convex set-valued maps, J. Math. Anal. Appl. 170 (1992), 477-500. MR 94a:54103

[BI] H. Ben-El-Mechaiekh and A. Idzik, A Leray-Schauder type theorem for approximable maps, Proc. Amer. Math. Soc. 122 (1994), 105-109. MR 94k:54074

[Br] C. Berge, Espaces Topologique, Dunod, Paris, 1959. MR 21:4401

[BK] G. D. Birkhoff and O. D. Kellogg, Invariant points in function space, Trans. Amer. Math. Soc. 23 (1922), 96-115.

[K] V. Klee, Shrinkable neighborhoods in Hausdorff linear spaces, Math. Ann. 141 (1960), 281-285. MR 24:A1003

[P1] Sehie Park, Some coincidence theorems on acyclic multifunctions and applications to KKM theory, Fixed Point Theory and Applications (K.-K.Tan, Ed.), World Sci., River Edge, NJ, 1992, pp.248-277. MR 93j:47087

[P2] Coincidences of composites of admissible u.s.c. maps and applications, Math. Rep. Acad. Sci. Canada 15 (1993), 125-130. MR 94f:47071

[P3] Some existence theorems for two variable functions on topological vector spaces, Kangweon-Kyungki Math. J. 3 (1995), 11-16. 
[P4] Generalized Leray-Schauder principles for compact admissible multifunctions, Top. Meth. Nonlinear Anal. 5 (1995), 271-277.

[PC] S. Park and M.-P. Chen, Generalized quasi-variational inequalities, Far East J. Math. Sci. 3 (1995), 199-204.

[PSW] S. Park, S. P. Singh, and B. Watson, Some fixed point theorems for composites of acyclic maps, Proc. Amer. Math. Soc. 121 (1994), 1151-1158. MR 94j:47089

[Pe] J.-P. Penot, Fixed point theorems without convexity, Bull. Soc. Math. France Mém. 60 (1979), 129-152. MR 81c:47061

[Sc1] H. Schaefer, Über die Methode der a priori Schranken, Math. Ann. 129 (1955), 415-416. MR 17:175

[Sc2] - Neue Existenzsätze in der Theorie nichtlinearer Integralgleichungen, Ber. Verh. Sächs. Akad. Wiss. Leipzig Math.-Natur. Kl. 101 (1955), no.7, 40pp. MR 20:1184

[Sö] R. Schöneberg, Leray-Schauder principle for condensing multi-valued mappings in topological linear spaces, Proc. Amer. Math. Soc. 72 (1978), 268-270. MR 81b:47075

[T] W. Takahashi, Existence theorems generalizing fixed point theorems for multivalued mappings, Fixed Point Theory and Applications (M.-A. Théra and J.-B. Baillon, Eds), Longman Sci. \& Tech., Essex, 1991, pp.397-406. MR 92m:54078

Department of Mathematics, Seoul National University, Seoul 151-742, Korea

E-mail address: shpark@math.snu.ac.kr 